\title{
A FACTOR ANALYSIS STUDY OF THE MAJOR ELEMENT GEOCHEMISTRY OF GRANITIC ROCKS
}

\author{
RISTO PIISPANEN and TUOMO ALAPIETI
}

\begin{abstract}
PIISPANEN, R. and ALAPIETI, T., 1977: A factor analysis study of the major element geochemistry of granitic rocks. Bull. Geol. Soc. Finland 49 (2): 143-150.

A Varimax-rotated R-mode factor analysis was performed on a collection of 195 whole-rock major element chemical analyses of granitic rocks, mainly Finnish. Factor loadings with corresponding eigenvalues and communalities were computed separately for 3 to 6 factors. The rapakivis, the non-rapakivis and the combined group of all the granitic rocks were each studied individually. The results show that, in contrast to the other granites, the rapakivis form a fairly homogeneous group with pronouncedly high mutual correlations between the components. A factor analysis, therefore, reveals one single factor with a very high eigenvalue. This factor is designated the femic factor on the basis of the most important participating components. In other granitic rocks three main factors emerge; these are termed the femic, the one-alkali and the biotite factor, respectively. The existence of the one-alkali factor reflects the even stronger negative correlation of $\mathrm{Na}_{2} \mathrm{O}$ and $\mathrm{K}_{2} \mathrm{O}$ in the nonrapakivis compared with the rapakivis.
\end{abstract}

Risto Piispanen and Tuomo Alapieti, Department of Geology, University of Oulu, SF-90100 Oulu 10, Finland.

\section{Introduction}

Since its early origins in psychology (see Koch and Link 1971, p. 121), factor analysis has been performed on collections of multivariate data within a variety of scientific fields. In geology applications of the method are already commonplace in palaeontology (e.g. Gould 1967), sedimentology (e.g. Krumbein and Graybill 1965; Imbrie and Purdy 1962), mineralogy (e.g. Saxena 1969) and economic geology (e.g. Koch and Link 1971), but in petrology and lithogeochemistry the applications to date are very few.
It is the aim of any factor analysis to try to find simple structure among a multitude of variables, and consequently to minimize the number of independent variables in terms of which the wealth of information is presented. A factor analysis thus creates new "artificial» properties which should cover to as great a degree as possible the total variances of the original properties. As a result of a successful factor analysis, for instance, information can be graphically displayed far more easily than would otherwise be possible, and the comprehensibility of the information contained in the data collection is thereby 
increased. On the other hand, the new artificial variables created in association with the analysis are often difficult to interpret empirically in physical terms and their real nature and associated geological implications often prove to be interpretable only with difficulty.

When applying factor analysis to a collection of major element data of a rock-group the main interest is directed towards a possible parallelism of the mineralogical composition and the factor pattern. In a simple hypothetical system, in which each chemical component would have a mineralogical counterpart of its own, a high correlation between some of the components would induce a correspondingly high correlation among some of the mineral phases and, in the analysis therefore, a factor could be created which would represent both the chemical components and the minerals and could also be interpreted in terms of the mineral composition. In real rocks in which most of the chemical components occupy positions in more than one mineral's lattice and, furthermore, each mineral (with rare exceptions) comprises more than one chemical component, the situation is much more complicated. High correlations among the chemical components may exist but the factors that can be created on the basis of the correlations may not be easily interpretable in terms of the mineral composition. To see whether the mineral composition is reflected in the factor pattern is one of the interesting points of lithogeochemical factor analysis.

The number of factors that can be created in a factor analysis is dependent on the degree of correlation between the properties to be studied, in this case the chemical components. Similarly, the resulting eigenvalues and the communalities also reflect the degree of correlation and the successfulness of the analysis. In a closed system such as a wholerock chemical analysis, i.e., in a system with a fixed sum (or nearly so) some apparent correlation resulting from arithmetical reasons can be of utmost importance. This type of correlation has been called induced correlation, and, Chayes in particular, has paid attention to it (Chayes 1962, Chayes and Kruskal 1966, Koch and Link 1971, p. 169). Therefore one of the interesting aspects of a factor analysis is also to study whether the induced correlation will manifest itself in the results of a lithogeochemical factor analysis.

\section{Material, method and results}

The collection of silicate analyses of granitic rocks that have been earlier described in detail by one of the authors (Piispanen 1977) was utilized as the basic data collection for the present study. For the data sources and other details the reader is referred to this earlier publication (Piispanen op. cit.). The collection consists of 195 analyses of which 40 represent rapakivis so that for the analyses three groups can be delimited, the total family of granitic rocks including the rapakivis, the rapakivis on their own and the total family excluding the rapakivis, the socalled non-rapakivi group.

The necessary computations were carried out on the University of Oulu computer system. The principle and the method of computation were based on the correlation matrix from which, as a first phase, a noniterative principal axes solution was calculated. Each of the three subgroups of the data collection were thereafter individually subjected to a Varimax-rotated factor analysis in which pertinent loadings, eigenvalues and communalities were calculated by turns for 3 to 6 factors. The results of the relevant cases are shown in Table 1.

In Table 2 the results are summarized and converted into chemical components. For each individual factor the components listed are those which contribute most strongly to 
Table 1. Factor loadings for individual runs covering 3 to 6 factors. A stands for the rapakivis, C for all granites $(C=A+B)$ and $B$ for the non-rapakivis $(B=C-A)$.

\begin{tabular}{|c|c|c|c|c|c|}
\hline & \multicolumn{3}{|c|}{ A } & & \multirow{2}{*}{$\begin{array}{c}\text { Commu- } \\
\text { nality }\end{array}$} \\
\hline & I & II & III & & \\
\hline $\mathrm{SiO}_{2}$ & -.759 & -.268 & -.566 & & .968 \\
\hline $\mathrm{TiO}_{2}$ & .826 & .210 & .247 & & .787 \\
\hline $\mathrm{Al}_{2} \mathrm{O}_{3}$ & .190 & -.023 & .830 & & .726 \\
\hline $\mathrm{Fe}_{2} \mathrm{O}_{3}$ & .499 & .731 & -.010 & & .784 \\
\hline $\mathrm{FeO}$ & .942 & .030 & .189 & & .924 \\
\hline $\mathrm{MnO}$ & .847 & -.019 & .190 & & .754 \\
\hline $\mathrm{MgO}$ & .554 & .570 & .318 & & .733 \\
\hline $\mathrm{CaO}$ & .842 & .025 & .375 & & .850 \\
\hline $\mathrm{Na}_{2} \mathrm{O}$ & .314 & -.662 & .071 & & .543 \\
\hline $\mathrm{K}_{2} \mathrm{O}$ & -.720 & .313 & .217 & & .663 \\
\hline $\mathrm{P}_{2} \mathrm{O}_{5}$ & .609 & .351 & .463 & & .709 \\
\hline $\mathrm{H}_{2} \mathrm{O}^{+}$ & -.086 & .768 & -.008 & & .597 \\
\hline \multirow[t]{4}{*}{$\mathrm{H}_{2} \mathrm{O}-$} & .173 & .640 & .180 & & .471 \\
\hline & 5.190 & 2.637 & 1.683 & & 9.509 \\
\hline & \multicolumn{4}{|c|}{ A } & \multirow{2}{*}{$\begin{array}{l}\text { Commu- } \\
\text { nality }\end{array}$} \\
\hline & I & II & III & IV & \\
\hline $\mathrm{SiO}_{2}$ & -.748 & -.292 & -.581 & -.002 & .982 \\
\hline $\mathrm{TiO}_{2}$ & -.795 & .238 & .245 & .232 & .803 \\
\hline $\mathrm{Al}_{2} \mathrm{O}_{3}$ & .176 & -.011 & .833 & .044 & .728 \\
\hline $\mathrm{Fe}_{2} \mathrm{O}_{3}$ & .492 & .742 & .000 & -.092 & .801 \\
\hline $\mathrm{FeO}$ & .945 & .057 & .209 & -.007 & .940 \\
\hline $\mathrm{MnO}$ & .829 & .008 & .194 & .190 & .761 \\
\hline $\mathrm{MgO}$ & .522 & .590 & .314 & .140 & .738 \\
\hline $\mathrm{CaO}$ & .862 & .047 & .408 & -.188 & .947 \\
\hline $\mathrm{Na}_{2} \mathrm{O}$ & .300 & -.648 & .061 & .307 & .607 \\
\hline $\mathrm{K}_{2} \mathrm{O}$ & -.720 & .293 & .209 & -.135 & .667 \\
\hline $\mathrm{P}_{2} \mathrm{O}_{5}$ & .552 & .378 & .442 & .401 & .805 \\
\hline $\mathrm{H}_{2} \mathrm{O}^{+}$ & -.115 & .767 & -.022 & .062 & .605 \\
\hline \multirow[t]{2}{*}{$\mathrm{H}_{2} \mathrm{O}^{-}$} & .136 & .648 & .166 & .152 & .490 \\
\hline & 5.007 & 2.704 & 1.709 & .455 & 9.874 \\
\hline
\end{tabular}

Table 1. cont.

\begin{tabular}{|c|c|c|c|c|c|c|}
\hline & \multicolumn{5}{|c|}{ A } & \multirow{2}{*}{$\begin{array}{c}\text { Commu- } \\
\text { nality }\end{array}$} \\
\hline & I & II & III & IV & $\mathrm{V}$ & \\
\hline $\mathrm{SiO}_{2}$ & -.761 & -.243 & -.572 & -.101 & -.085 & .982 \\
\hline $\mathrm{TiO}_{2}$ & .767 & .161 & .246 & .396 & .020 & .832 \\
\hline $\mathrm{Al}_{2} \mathrm{O}_{5}$ & .180 & -.041 & .831 & .056 & .046 & .730 \\
\hline $\mathrm{Fe}_{2} \mathrm{O}_{3}$ & .519 & .725 & .008 & .088 & -.002 & .802 \\
\hline $\mathrm{FeO}$ & .948 & .009 & .189 & .057 & .097 & .948 \\
\hline $\mathrm{MnO}$ & .822 & -.065 & .155 & .091 & .375 & .852 \\
\hline MgO & .515 & .533 & .329 & .353 & -.028 & .783 \\
\hline $\mathrm{CaO}$ & .883 & .027 & .399 & -.060 & -.065 & .947 \\
\hline $\mathrm{Na}_{2} \mathrm{O}$ & .246 & -.701 & .042 & .208 & .103 & .607 \\
\hline $\mathrm{K}_{2} \mathrm{O}$ & -.681 & .337 & .215 & -.234 & .084 & .686 \\
\hline $\mathrm{P}_{2} \mathrm{O}_{5}$ & .523 & .279 & .434 & .449 & .253 & .806 \\
\hline $\mathrm{H}_{2} \mathrm{O}^{+}$ & -.101 & .754 & -.004 & .171 & .043 & .610 \\
\hline $\mathrm{H}_{2} \mathrm{O}-$ & .157 & .605 & .147 & .068 & .400 & .577 \\
\hline & 4.922 & 2.518 & 1.663 & .651 & .408 & 10.161 \\
\hline
\end{tabular}




\begin{tabular}{|c|c|c|c|c|c|c|c|}
\hline & \multicolumn{6}{|c|}{ A } & \multirow{2}{*}{$\begin{array}{c}\text { Commu- } \\
\text { nality }\end{array}$} \\
\hline & I & II & III & IV & $\mathrm{V}$ & VI & \\
\hline $\mathrm{SiO}_{2}$ & -.751 & -.269 & -.569 & -.151 & -.061 & -.003 & .986 \\
\hline $\mathrm{TiO}_{2}$ & .746 & .194 & .240 & .377 & -.001 & .197 & .832 \\
\hline $\mathrm{Al}_{2} \mathrm{O}_{3}$ & .177 & -.023 & .832 & .084 & .034 & .006 & .732 \\
\hline $\mathrm{Fe}_{2} \mathrm{O}_{3}$ & .504 & .698 & -.014 & .193 & -.039 & -.149 & .802 \\
\hline $\mathrm{FeO}$ & .941 & .041 & .191 & .089 & .112 & .056 & .948 \\
\hline $\mathrm{MnO}$ & .798 & -.029 & .145 & .167 & .444 & .055 & .887 \\
\hline $\mathrm{MgO}$ & .481 & .474 & .281 & .528 & .012 & -.104 & .825 \\
\hline $\mathrm{CaO}$ & .887 & .028 & .397 & .018 & -.047 & -.065 & .952 \\
\hline $\mathrm{Na}_{2} \mathrm{O}$ & .249 & -.597 & .080 & -.004 & .088 & .434 & .621 \\
\hline $\mathrm{K}_{2} \mathrm{O}$ & -.683 & .275 & .192 & -.093 & .100 & -.324 & .703 \\
\hline $\mathrm{P}_{2} \mathrm{O}_{5}$ & .489 & .333 & .427 & .430 & .216 & .210 & .807 \\
\hline $\mathrm{H}_{2} \mathrm{O}^{+}$ & .112 & .774 & -.003 & .123 & -.089 & .010 & .635 \\
\hline \multirow[t]{2}{*}{$\mathrm{H}_{2} \mathrm{O}-$} & .143 & .688 & .165 & -.005 & .271 & .074 & .599 \\
\hline & 4.748 & 2.440 & 1.620 & .733 & .364 & .426 & 10.330 \\
\hline
\end{tabular}

Table 1. cont.

\begin{tabular}{|c|c|c|c|c|c|}
\hline & \multicolumn{3}{|c|}{ B } & & \multirow{2}{*}{$\begin{array}{c}\text { Commu- } \\
\text { nality }\end{array}$} \\
\hline & I & II & III & & \\
\hline $\mathrm{SiO}_{2}$ & -.820 & .236 & -.211 & & .772 \\
\hline $\mathrm{TiO}_{2}$ & .678 & .062 & .468 & & .683 \\
\hline $\mathrm{Al}_{2} \mathrm{O}_{3}$ & .392 & -.552 & -.228 & & .511 \\
\hline $\mathrm{Fe}_{2} \mathrm{O}_{2}$ & .231 & .098 & .718 & & .579 \\
\hline $\mathrm{FeO}$ & .802 & .045 & .073 & & .651 \\
\hline $\mathrm{MnO}$ & .102 & -.085 & .554 & & .325 \\
\hline $\mathrm{MgO}$ & .826 & -.174 & .162 & & .739 \\
\hline $\mathrm{CaO}$ & .674 & -.488 & -.055 & & .695 \\
\hline $\mathrm{Na}_{2} \mathrm{O}$ & -.107 & -.736 & .264 & & .623 \\
\hline $\mathrm{K}_{2} \mathrm{O}$ & -.356 & .707 & -.081 & & .634 \\
\hline $\mathrm{P}_{2} \mathrm{O}_{5}$ & .481 & -.026 & .149 & & .255 \\
\hline $\mathrm{H}_{2} \mathrm{O}^{+}$ & .664 & .102 & .138 & & .471 \\
\hline \multirow[t]{4}{*}{$\mathrm{H}_{2} \mathrm{O}^{-}$} & .121 & .231 & .095 & & .077 \\
\hline & 3.956 & 1.758 & 1.300 & & 7.014 \\
\hline & \multicolumn{4}{|c|}{ B } & \multirow{2}{*}{$\begin{array}{c}\text { Commu- } \\
\text { nality }\end{array}$} \\
\hline & I & II & III & IV & \\
\hline $\mathrm{SiO}_{2}$ & -.550 & .059 & -.349 & -.664 & .868 \\
\hline $\mathrm{TiO}_{2}$ & .660 & -.083 & .469 & .147 & .684 \\
\hline $\mathrm{Al}_{2} \mathrm{O}_{3}$ & .052 & .175 & -.055 & .796 & .670 \\
\hline $\mathrm{Fe}_{2} \mathrm{O}_{3}$ & .226 & -.083 & .734 & -.037 & .598 \\
\hline FeO & .704 & -.164 & .112 & .341 & .651 \\
\hline MnO & .065 & .061 & .582 & .051 & .349 \\
\hline $\mathrm{MgO}$ & .769 & .091 & .164 & .359 & .755 \\
\hline $\mathrm{CaO}$ & .461 & .238 & .033 & .661 & .707 \\
\hline $\mathrm{Na}_{2} \mathrm{O}$ & -.098 & .733 & .229 & .188 & .635 \\
\hline $\mathrm{K}_{2} \mathrm{O}$ & -.387 & -.707 & -.012 & -.300 & .740 \\
\hline $\mathrm{P}_{2} \mathrm{O}_{5}$ & .543 & .077 & .093 & .047 & .312 \\
\hline $\mathrm{H}_{2} \mathrm{O}^{+}$ & .748 & -.031 & .068 & .026 & .566 \\
\hline \multirow[t]{2}{*}{$\mathrm{H}_{2} \mathrm{O}^{-}$} & .058 & -.286 & .146 & .030 & .107 \\
\hline & 3.111 & 1.270 & 1.350 & 1.912 & 7.643 \\
\hline
\end{tabular}


Table 1. cont.

\begin{tabular}{|c|c|c|c|c|c|c|c|}
\hline & \multicolumn{5}{|c|}{ B } & & \multirow{2}{*}{$\begin{array}{c}\text { Commu } \\
\text { nality }\end{array}$} \\
\hline & $\mathrm{I}$ & II & III & IV & $\mathrm{V}$ & & \\
\hline $\mathrm{SiO}_{2}$ & -.515 & .065 & -.332 & -.674 & .217 & & .881 \\
\hline $\mathrm{TiO}_{2}$ & .643 & -.085 & .458 & .162 & -.178 & & .688 \\
\hline $\mathrm{AJ}_{2} \mathrm{O}_{3}$ & .067 & .145 & -.085 & .823 & .006 & & .709 \\
\hline $\mathrm{Fe}_{2} \mathrm{O}_{3}$ & .278 & -.129 & .707 & -.000 & .086 & & .602 \\
\hline $\mathrm{FeO}$ & .502 & -.060 & .182 & .256 & -.617 & & .735 \\
\hline $\mathrm{MnO}$ & -.005 & .079 & .625 & .018 & -.131 & & .414 \\
\hline $\mathrm{MgO}$ & .668 & .137 & .189 & .337 & -.378 & & .757 \\
\hline $\mathrm{CaO}$ & .310 & .302 & .087 & .609 & -.416 & & .738 \\
\hline $\mathrm{Na}_{2} \mathrm{O}$ & .002 & .660 & .204 & .259 & .343 & & .662 \\
\hline $\mathrm{K}_{2} \mathrm{O}$ & -.297 & -.746 & -.066 & -.281 & .188 & & .763 \\
\hline $\mathrm{P}_{2} \mathrm{O}_{5}$ & .602 & .047 & .051 & .100 & .019 & & .377 \\
\hline $\mathrm{H}_{2} \mathrm{O}^{+}$ & .757 & -.030 & .043 & .055 & -.145 & & .601 \\
\hline \multirow[t]{4}{*}{$\mathrm{H}_{2} \mathrm{O}^{-}$} & .092 & -.307 & .116 & .046 & .024 & & .119 \\
\hline & 2.588 & 1.258 & 1.358 & 1.868 & .976 & & 8.047 \\
\hline & \multicolumn{4}{|c|}{ B } & & & \multirow{2}{*}{$\begin{array}{l}\text { Commu- } \\
\text { nality }\end{array}$} \\
\hline & I & II & III & IV & $\mathrm{V}$ & VI & \\
\hline $\mathrm{SiO}_{2}$ & -.528 & -.031 & -.326 & -.634 & -.288 & -.123 & .886 \\
\hline $\mathrm{TiO}_{2}$ & .654 & -.015 & .450 & .133 & .172 & .117 & .691 \\
\hline $\mathrm{Al}_{2} \mathrm{O}_{3}$ & .072 & .214 & -.086 & .800 & .142 & -.054 & .721 \\
\hline $\mathrm{Fe}_{2} \mathrm{O}_{3}$ & .270 & .063 & .665 & -.025 & -.072 & .293 & .611 \\
\hline $\mathrm{FeO}$ & .524 & -.202 & .213 & .203 & .580 & -.022 & .739 \\
\hline $\mathrm{MnO}$ & .014 & .066 & .639 & .009 & .113 & -.065 & .431 \\
\hline $\mathrm{MgO}$ & .665 & .102 & .177 & .257 & .455 & .014 & .757 \\
\hline $\mathrm{CaO}$ & .274 & .274 & .041 & .469 & .627 & .098 & .775 \\
\hline $\mathrm{Na}_{2} \mathrm{O}$ & -.035 & .756 & .143 & .191 & -.081 & -.166 & .663 \\
\hline $\mathrm{K}_{2} \mathrm{O}$ & -.240 & -.679 & .002 & -.123 & -.482 & .129 & .782 \\
\hline $\mathrm{P}_{2} \mathrm{O}_{5}$ & .581 & .143 & .009 & .055 & .054 & .136 & .383 \\
\hline $\mathrm{H}_{2} \mathrm{O}^{+}$ & .772 & -.034 & .051 & .050 & .118 & -.037 & .618 \\
\hline \multirow[t]{2}{*}{$\mathrm{H}_{2} \mathrm{O}-$} & .055 & -.126 & .053 & .000 & .009 & .496 & .268 \\
\hline & 2.572 & 1.250 & 1.272 & 1.444 & 1.344 & .442 & 8.326 \\
\hline
\end{tabular}

Table 1. cont.

\begin{tabular}{|c|c|c|c|c|}
\hline & \multicolumn{3}{|c|}{$\mathrm{C}$} & \multirow{2}{*}{$\begin{array}{c}\text { Commu- } \\
\text { nality }\end{array}$} \\
\hline & I & II & III & \\
\hline $\mathrm{SiO}_{2}$ & -.842 & .138 & -.263 & .797 \\
\hline $\mathrm{TiO}_{2}$ & .655 & .093 & .519 & .708 \\
\hline $\mathrm{Al}_{2} \mathrm{O}_{3}$ & .449 & -.494 & $\begin{array}{r}.239 \\
\end{array}$ & .502 \\
\hline $\mathrm{Fe}_{2} \mathrm{O}_{3}$ & .240 & .105 & .702 & .561 \\
\hline $\mathrm{FeO}$ & .739 & .157 & .234 & .625 \\
\hline $\mathrm{MnO}$ & .136 & -.096 & .576 & .359 \\
\hline $\mathrm{MgO}$ & .740 & -.247 & .155 & .632 \\
\hline $\mathrm{CaO}$ & .732 & -.409 & .008 & .703 \\
\hline $\mathrm{Na}_{2} \mathrm{O}$ & -.092 & $\begin{array}{r}.799 \\
-\end{array}$ & .159 & .673 \\
\hline $\mathrm{K}_{2} \mathrm{O}$ & -.329 & .728 & -.047 & .641 \\
\hline $\mathrm{P}_{2} \mathrm{O}_{5}$ & .521 & -.021 & .198 & .311 \\
\hline $\mathrm{H}_{2} \mathrm{O}^{+}$ & 563 & .113 & .135 & .348 \\
\hline \multirow[t]{2}{*}{$\mathrm{H}_{2} \mathrm{O}^{-}$} & .173 & .286 & .143 & .132 \\
\hline & 3.780 & 1.808 & 1.403 & 6.991 \\
\hline
\end{tabular}


Table 1 - continued

\begin{tabular}{|c|c|c|c|c|c|c|c|}
\hline & \multicolumn{4}{|c|}{$\mathrm{C}$} & & & \multirow{2}{*}{$\begin{array}{c}\text { Commu- } \\
\text { nality }\end{array}$} \\
\hline & I & II & III & IV & & & \\
\hline $\mathrm{SiO}_{2}$ & -.718 & -.046 & -.399 & -.405 & & & .841 \\
\hline $\mathrm{TiO}_{2}$ & .330 & .153 & .568 & .503 & & & .708 \\
\hline $\mathrm{Al}_{2} \mathrm{O}_{3}$ & .672 & -.306 & -.119 & .032 & & & .560 \\
\hline $\mathrm{Fe}_{2} \mathrm{O}_{3}$ & -.041 & .065 & .689 & .285 & & & .562 \\
\hline $\mathrm{FeO}$ & .566 & .313 & .367 & .349 & & & .675 \\
\hline $\mathrm{MnO}$ & .126 & -.066 & .630 & -.022 & & & .417 \\
\hline $\mathrm{MgO}$ & .446 & -.177 & .159 & .664 & & & .696 \\
\hline $\mathrm{CaO}$ & .777 & -.207 & .140 & .280 & & & .745 \\
\hline $\mathrm{Na}_{2} \mathrm{O}$ & .095 & -.809 & .113 & -.044 & & & .679 \\
\hline $\mathrm{K}_{2} \mathrm{O}$ & -.345 & .684 & -.020 & -.305 & & & .680 \\
\hline $\mathrm{P}_{2} \mathrm{O}_{5}$ & .238 & .008 & .194 & .504 & & & .348 \\
\hline $\mathrm{H}_{2} \mathrm{O}^{+}$ & .076 & .075 & .058 & .762 & & & .596 \\
\hline \multirow[t]{4}{*}{$\mathrm{H}_{2} \mathrm{O}^{-}$} & .018 & .293 & .164 & .139 & & & .132 \\
\hline & 2.408 & 1.514 & 1.628 & 2.091 & & & 7.640 \\
\hline & \multicolumn{5}{|c|}{$\mathrm{C}$} & & \multirow{2}{*}{$\begin{array}{c}\text { Commu- } \\
\text { nality }\end{array}$} \\
\hline & I & II & III & IV & $\mathrm{V}$ & & \\
\hline $\mathrm{SiO}_{2}$ & -.611 & .061 & -.388 & -.447 & -.386 & & .876 \\
\hline $\mathrm{TiO}_{2}$ & .119 & -.076 & .521 & .507 & .404 & & .712 \\
\hline $\mathrm{Al}_{2} \mathrm{O}_{3}$ & .790 & .183 & -.069 & .087 & .032 & & .671 \\
\hline $\mathrm{Fe}_{2} \mathrm{O}_{3}$ & -.011 & -.133 & .699 & .305 & -.022 & & .600 \\
\hline $\mathrm{FeO}$ & .168 & -.107 & .271 & .336 & .741 & & .775 \\
\hline $\mathrm{MnO}$ & .023 & .102 & .610 & -.023 & .205 & & .425 \\
\hline MgO & .319 & .220 & .141 & .674 & .268 & & .696 \\
\hline $\mathrm{CaO}$ & .579 & .288 & .109 & .296 & .489 & & .756 \\
\hline $\mathrm{Na}_{2} \mathrm{O}$ & .280 & .703 & .179 & -.030 & -.285 & & .686 \\
\hline $\mathrm{K}_{2} \mathrm{O}$ & -.260 & -.742 & -.022 & -.296 & -.124 & & .726 \\
\hline $\mathrm{P}_{2} \mathrm{O}_{5}$ & .153 & .012 & .178 & .514 & .175 & & .349 \\
\hline $\mathrm{H}_{2} \mathrm{O}^{+}$ & .024 & -.069 & .046 & .768 & .063 & & .602 \\
\hline \multirow[t]{4}{*}{$\mathrm{H}_{2} \mathrm{O}-$} & .077 & -.368 & .175 & .164 & -.037 & & .200 \\
\hline & 1.657 & 1.398 & 1.489 & 2.183 & 1.348 & & 8.075 \\
\hline & \multicolumn{6}{|c|}{$\mathrm{C}$} & \multirow{2}{*}{$\begin{array}{c}\text { Commu- } \\
\text { nality }\end{array}$} \\
\hline & I & II & III & IV & $\mathrm{V}$ & VI & \\
\hline $\mathrm{SiO}_{2}$ & -.610 & .064 & -.378 & -.308 & -.409 & -.307 & .876 \\
\hline $\mathrm{TiO}_{2}$ & .113 & -.064 & .491 & .326 & .418 & .445 & .736 \\
\hline $\mathrm{Al}_{2} \mathrm{O}_{3}$ & .789 & .182 & -.077 & .043 & .041 & .084 & .673 \\
\hline $\mathrm{Fe}_{2} \mathrm{O}_{3}$ & -.002 & -.134 & .723 & .267 & .009 & .096 & .621 \\
\hline $\mathrm{FeO}$ & .163 & -.120 & .254 & .205 & .752 & .251 & .776 \\
\hline $\mathrm{MnO}$ & .019 & .107 & .599 & -.078 & .214 & .058 & .426 \\
\hline MgO & .339 & .194 & .170 & .631 & .324 & .169 & .713 \\
\hline $\mathrm{CaO}$ & .588 & .259 & .123 & .261 & .527 & .033 & .775 \\
\hline $\mathrm{Na}_{2} \mathrm{O}$ & .278 & .722 & .157 & -.042 & -.269 & .058 & .701 \\
\hline $\mathrm{K}_{2} \mathrm{O}$ & -.286 & -.721 & -.040 & -.322 & -.176 & .029 & .738 \\
\hline $\mathrm{P}_{2} \mathrm{O}_{5}$ & .139 & .042 & .124 & .311 & .171 & .563 & .479 \\
\hline $\mathrm{H}_{2} \mathrm{O}^{+}$ & .043 & -.085 & .079 & .725 & .110 & .235 & .608 \\
\hline \multirow[t]{2}{*}{$\mathrm{H}_{2} \mathrm{O}^{-}$} & .084 & -.375 & .200 & .163 & -.027 & .010 & .215 \\
\hline & 1.683 & 1.380 & 1.468 & 1.542 & 1.483 & .781 & 8.338 \\
\hline
\end{tabular}


Table 2. Summary of data from Table 1 converted into symbols for the most significant chemical components contributing to the generation of each individual factor. For the meaning of A, B, and C see Table 1. Cases from 3 to 6 factors are presented.

\begin{tabular}{|c|c|c|c|}
\hline & A & B & $\mathrm{C}$ \\
\hline I & $\begin{array}{l}-\mathrm{Si}, \mathrm{Ti}, \mathrm{Fe}^{3+}, \mathrm{Fe}^{2+}, \\
\mathrm{Mn}, \mathrm{Mg}, \mathrm{Ca},-\mathrm{K}, \mathrm{P}\end{array}$ & $\begin{array}{l}-\mathrm{Si}, \mathrm{Ti},(\mathrm{Al}), \mathrm{Fe}^{2+} \\
\mathrm{Mg}, \mathrm{Ca}, \mathrm{P}, \mathrm{H}_{2} \mathrm{O}^{+}\end{array}$ & $\begin{array}{l}-\mathrm{Si}, \mathrm{Ti}, \mathrm{Al}, \mathrm{Fe}^{2+}, \mathrm{Mg} \\
\mathrm{Ca}, \mathrm{P}, \mathrm{H}_{2} \mathrm{O}^{+}\end{array}$ \\
\hline II & $\mathrm{Fe}^{3+}, \mathrm{Mg},-\mathrm{Na}, \mathrm{H}_{2} \mathrm{O}^{+}, \mathrm{H}_{2} \mathrm{O}^{-}$ & $-\mathrm{Na}, \mathrm{K}, \mathrm{Al},(-\mathrm{Ca})$ & $-\mathrm{Al},-\mathrm{Ca},-\mathrm{Na}, \mathrm{K}$ \\
\hline III & $-\mathrm{Si}, \mathrm{Al}, \mathrm{P}$ & $\mathrm{Ti}, \mathrm{Fe}^{3+}, \mathrm{Mn}$ & $\mathrm{Ti}, \mathrm{Fe}^{3+}, \mathrm{Mn}$ \\
\hline I & $\begin{array}{l}-\mathrm{Si}, \mathrm{Ti}, \mathrm{Fe}^{3+}, \mathrm{Fe}^{2+}, \mathrm{Mn} \\
\mathrm{Mg}, \mathrm{Ca},-\mathrm{K}, \mathrm{P}\end{array}$ & $\begin{array}{l}-\mathrm{Si}, \mathrm{Ti}, \mathrm{Fe}^{2+}, \mathrm{Mg},(\mathrm{Ca}) \\
\mathrm{P}, \mathrm{H}_{2} \mathrm{O}^{+}\end{array}$ & $-\mathrm{Si}, \mathrm{Al}, \mathrm{Fe}^{2+}, \mathrm{Mg}, \mathrm{Ca}$ \\
\hline II & $\mathrm{Fe}^{3+}, \mathrm{Mg},-\mathrm{Na}, \mathrm{H}_{2} \mathrm{O}^{+}, \mathrm{H}_{2} \mathrm{O}^{-}$ & $\mathrm{Na},-\mathrm{K}$ & - Na, K \\
\hline III & - $\mathrm{Si}, \mathrm{Al}, \mathrm{Ca}, \mathrm{P}$ & $\mathrm{Ti}, \mathrm{Fe}^{3+}, \mathrm{Mn}$ & $\mathrm{Ti}, \mathrm{Fe}^{3+}, \mathrm{Mn}$ \\
\hline IV & $(\mathrm{Na}), \mathrm{P}$ & —Si, Al, Ca & $\mathrm{Ti}, \mathrm{Mg}, \mathrm{P}, \mathrm{H}_{2} \mathrm{O}^{+}$ \\
\hline I & $\begin{array}{l}-\mathrm{Si}, \mathrm{Ti}, \mathrm{Fe}^{3+}, \mathrm{Fe}^{2+}, \mathrm{Mn} \\
\mathrm{Mg}, \mathrm{Ca},-\mathrm{K}, \mathrm{P}\end{array}$ & $\begin{array}{l}-\mathrm{Si}, \mathrm{Ti}, \mathrm{Fe}^{2+}, \mathrm{Mg}, \mathrm{P}, \\
\mathrm{H}_{2} \mathrm{O}^{+}\end{array}$ & -Si, Al, Ca \\
\hline II & $\mathrm{Fe}^{3+}, \mathrm{Mg},-\mathrm{Na}, \mathrm{H}_{2} \mathrm{O}^{+}, \mathrm{H}_{2} \mathrm{O}^{-}$ & $\mathrm{Na}, \mathrm{K}$ & $\mathrm{Na},-\mathrm{K}$ \\
\hline III & $-\mathrm{Si}, \mathrm{Al}, \mathrm{P},(\mathrm{Ca})$ & $\mathrm{Ti}, \mathrm{Fe}^{3+}, \mathrm{Mn}$ & $\mathrm{Ti}, \mathrm{Fe}^{3+}, \mathrm{Mn}$ \\
\hline IV & $\mathrm{P},(\mathrm{Ti}),(\mathrm{Mg})$ & —Si, Al, Ca & $\mathrm{Ti}, \mathrm{Mg}, \mathrm{P}, \mathrm{H}_{2} \mathrm{O}^{+}$ \\
\hline V & $(\mathrm{Mn}), \mathrm{H}_{2} \mathrm{O}^{-}$ & $-\mathrm{Fe}^{2+},-\mathrm{Ca}$ & $\mathrm{Fe}^{2+}, \mathrm{Ca}$ \\
\hline I & $\begin{array}{l}-\mathrm{Si}, \mathrm{Ti}, \mathrm{Fe}^{3+}, \mathrm{Fe}^{2+}, \mathrm{Mn} \\
\mathrm{Mg}, \mathrm{Ca},-\mathrm{K}, \mathrm{P}\end{array}$ & $-\mathrm{Si}, \mathrm{Ti}, \mathrm{Fe}^{2+}, \mathrm{Mg}, \mathrm{P}, \mathrm{H}_{2} \mathrm{O}^{+}$ & $-\mathrm{Si}, \mathrm{Al}, \mathrm{Ca}$ \\
\hline II & $\mathrm{Fe}^{3+},(\mathrm{Mg}),-\mathrm{Na}, \mathrm{H}_{2} \mathrm{O}^{+}, \mathrm{H}_{2} \mathrm{O}^{-}$ & $\mathrm{Na},-\mathrm{K}$ & $\mathrm{Na},-\mathrm{K}$ \\
\hline III & —Si, Al, (Ca), P & $\mathrm{Ti}, \mathrm{Fe}^{3+}, \mathrm{Mn}$ & $\mathrm{Ti}, \mathrm{Fe}^{3+}, \mathrm{Mn}$ \\
\hline IV & $\mathrm{Mg}, \mathrm{P},(\mathrm{Ti})$ & 一 $\mathrm{Si}, \mathrm{Al}, \mathrm{Ca}$ & $\mathrm{Mg}, \mathrm{H}_{2} \mathrm{O}^{+}$ \\
\hline $\mathrm{V}$ & $\mathrm{Mn}$ & $\mathrm{Fe}^{2+}, \mathrm{Mg}, \mathrm{Ca},-\mathrm{K}$ & $\mathrm{Fe}^{2+}, \mathrm{Ca}$ \\
\hline VI & $\mathrm{Na},(-\mathrm{K})$ & $\mathrm{H}_{2} \mathrm{O}^{-}$ & $\mathrm{P},(\mathrm{Ti})$ \\
\hline
\end{tabular}

the generation of an individual factor. The listed components regularly have loadings of 0.4 or higher.

Both rapakivis and the total group of all the granitic rocks have, as their first factor, a factor in which $\mathrm{Fe}^{2+}, \mathrm{Mg}, \mathrm{Ca}$, Ti and negative silica have significant loadings. On the basis of the most important participating components the factor could, perhaps, be adequately called the femic factor. In rapakivis this factor has a very large eigenvalue (4.748 to 5.190) implying that a great deal of the variation of the chemical composition of the rapakivis can be described in terms of this single factor. The factor score pertai- ning to this factor for any rapakivi would reveal a great deal about the relationship of that particular rapakivi to the others.

In summary, the factor analysis among the rapakivis reveals one clear and prominent factor and some additional less clear and less significant factors. This situation is obviously a result of the homogeneity of the rock-group in comparison with the heterogeneity of the total group of granites.

In addition to the first, the femic factor, the rapakivis exhibit a second factor in which many of the same components which formed the first factor have large loadings. The most significant exception is $\mathrm{Na}_{2} \mathrm{O}$ which 
here makes its first appearance, potassium being already present under the first factor. The allocation of soda and potassium to different factors in the rapakivis is discussed in association with the factors for the total family of the granitic rocks.

The third factor for the rapakivis is a negative silica-alumina factor. In this the arithmetically induced negative correlation between the two largest chemical components is obviously the reason for the formation of the factor.

In contrast with the rapakivis, the total group of granites have a second factor that carries large loadings for both potassium and soda. This factor is a result of the strong (- 0.589) negative correlation of the components in the granites. Soda and potassium are also negatively correlated in the rapakivis, but the numerical value of the corre- lation (- -0.521$)$ there does not seem to be large enough to make the components fall under a common factor (in order to save space the correlation matrices are not reproduced here). A geological implication of these relationships is, perhaps, to indicate the ability of the rapakivi alkali feldspar to carry more soda either as perthite or antiperthite or as a homogeneous solid solution than that of the other granitic rocks.

As the third factor the total group of granites show, as a rule, a factor in which $\mathrm{Ti}$, ferric iron and $\mathrm{MnO}$ have large loadings. This factor does not make its appearance among the rapakivis. The factor is most easily interpreted as a biotite factor containing the femic elements of biotite and excluding $\mathrm{CaO}$ which falls under the first factor, the femic factor, where hornblende is probably the main mineral representative.

\section{REFERENCES}

Chayes, F. (1962) Numerical correlation and petrographic variation. J. Geol. 70: 440-452.

Chayes, F. and Kruskal, W. (1966) An approximate statistical test for correlations between proportions. J. Geol. 74: 692-702.

Gould, S. J. (1967) Evolutionary patterns in Pelycosaurian reptiles: a factor analytic study. Evolution, 21: 385-401.

Imbrie, J. and Purdy, E. G. (1962) Classification of modern Bahamian carbonate sediments. AAPG Symposium on Classification of Carbonate Rocks, Memoir 1: 253-272.

Koch, G. S. and Link, R. F. (1971) Statistical Analysis of Geological Data. Vol. 2. J. Wiley \&
Sons. New York.

Krumbein, W. C. and Graybill, F. A. (1965) An Introduction to Statistical Models in Geology. McGraw-Hill. New York.

Piispanen, R. (1977) Major element geochemistry of the granitic rocks. Bull. Geol. Soc. Finland 49 (2): $73-78$.

Saxena, S. K. (1969) Silicate solid solutions and geothermometry. 4. Statistical study of chemical data on garnets and clinopyroxene. Contr. Min. Petr. 23: 140-156.

Manuscript received 23 June, 1977 